Supporting Information for:

\title{
Model-Based Insight into Single-Molecule Plasmonic Mislocalization
}

Tiancheng Zuo ${ }^{1,+}$, Harrison J. Goldwynn ${ }^{2,+}$, David J. Masiello ${ }^{2, *}$, Julie S. Biteen ${ }^{1, *}$

${ }^{1}$ Department of Chemistry, University of Michigan, Ann Arbor, Michigan 48109, United States

${ }^{2}$ Department of Chemistry, University of Washington, Seattle, Washington 98195, United States

${ }^{+}$T.Z. and H.J.G. contributed equally to this work

*Correspondence: jsbiteen@umich.edu, masiello@uw.edu

\section{Contents:}

- Supporting Figures S1 - S14

- Supporting Table S1 

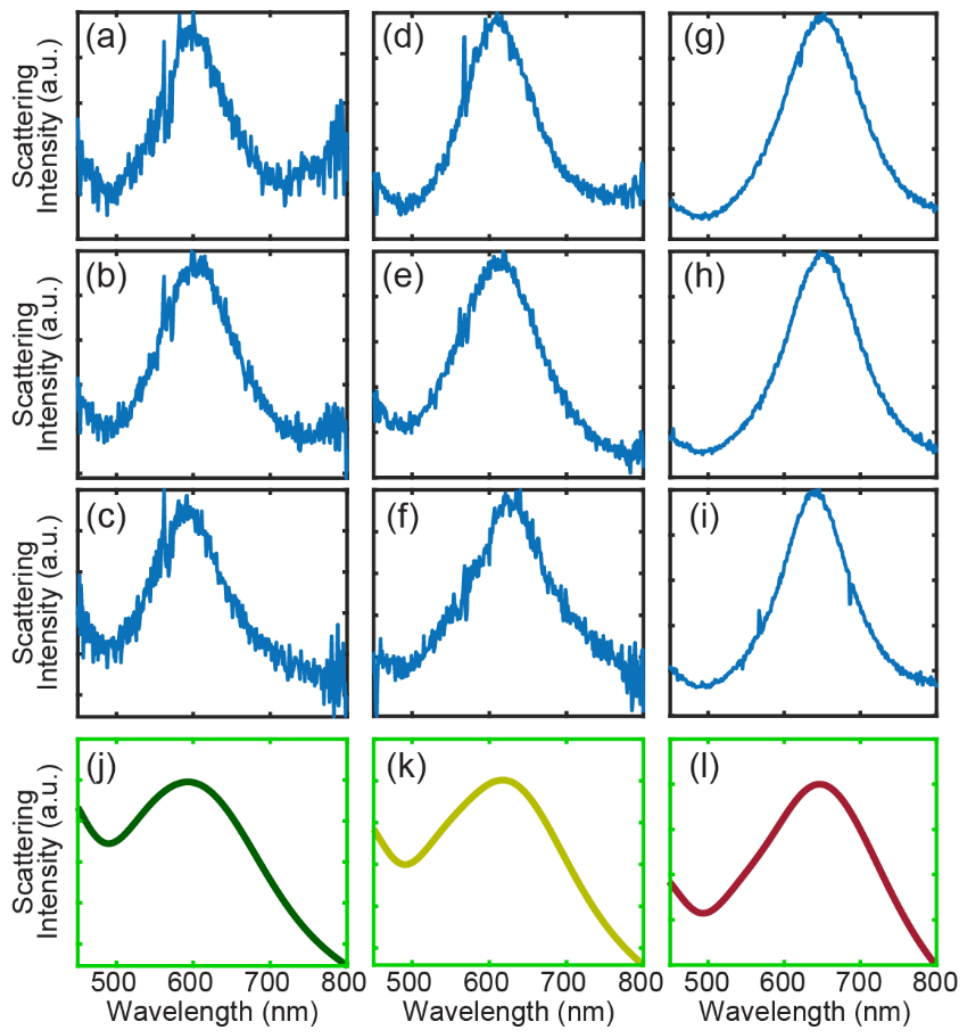

Figure S1. Experimental darkfield scattering spectra of three representative particles for each of three different nanodisk sizes: (a - c) 70-nm nanodisks; (d - f) 76-nm nanodisks; (g - i) 86-nm nanodisks. The scattering spectra in the top row correspond to the scattering spectra in Figure 2. $(\mathbf{j}-\mathbf{l})$ Calculated spectra determined by finite-difference time-domain simulation of the full sample geometry (Ansys Lumerical FDTD Solutions software package) for (j) 70-nm, (k) 76nm, and (l) 86-nm nanodisks. 

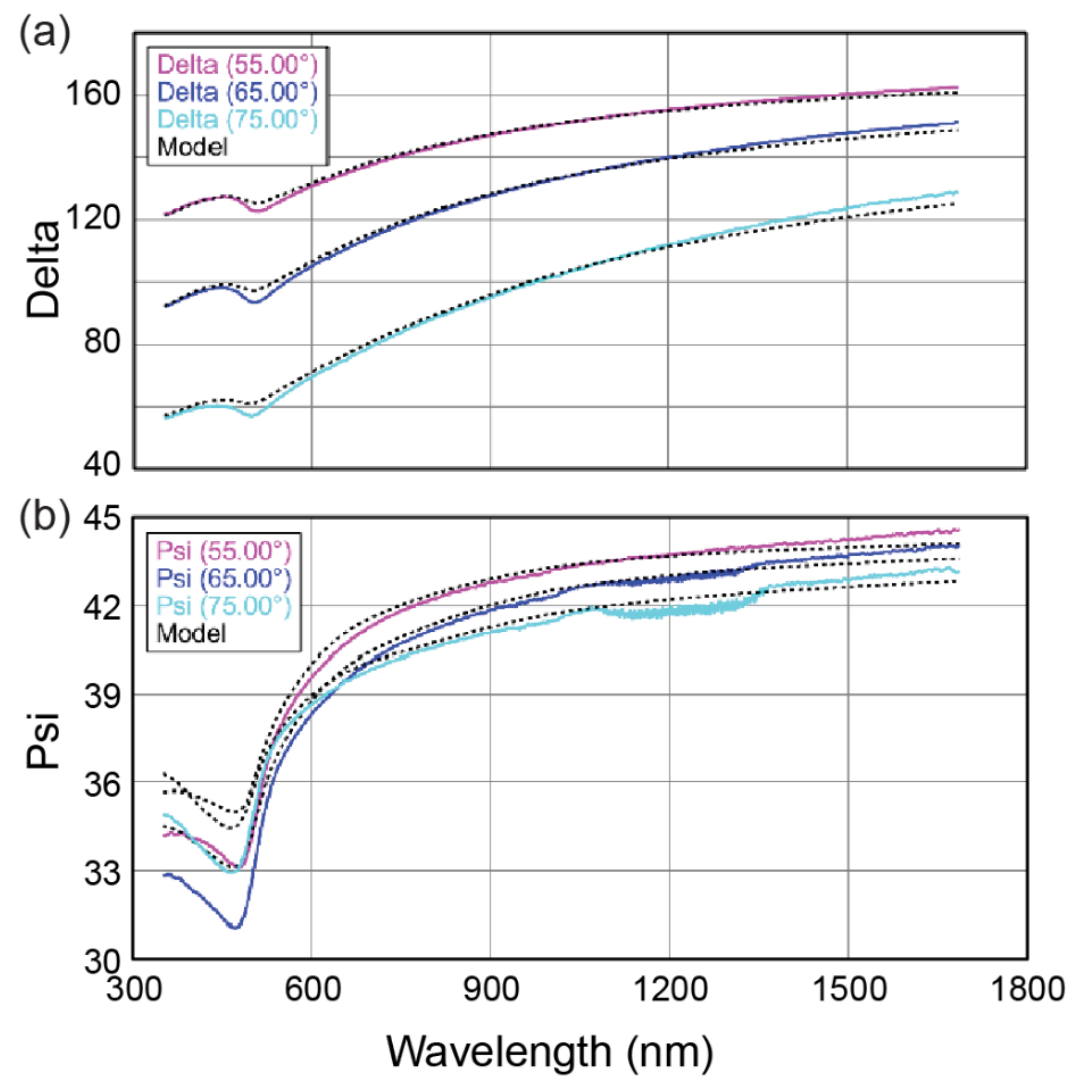

Figure S2. Variable angle spectroscopic ellipsometry (VASE) characterization of gold/ $/ \mathrm{SiO}_{2}$ films made under the same condition as the nanoparticles. 


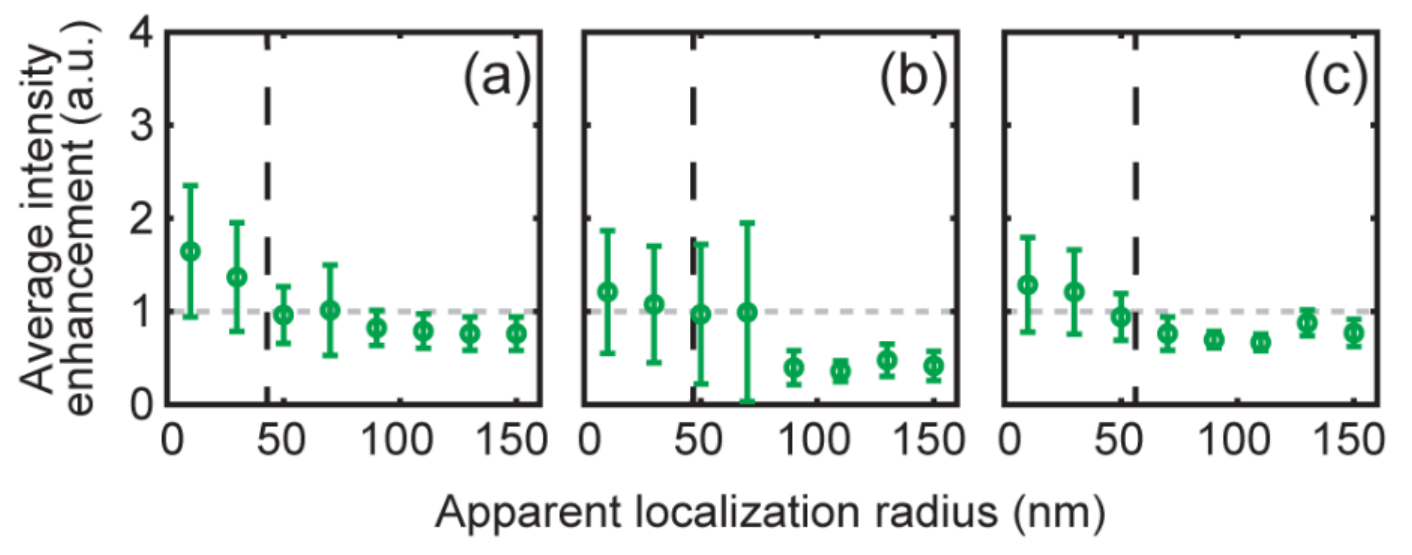

Figure S3. $(\mathbf{a}-\mathbf{c})$ Dependence of the fluorescence emission intensity on the apparent emission localization for ATTO590 dyes about 70-, 76-, and 86-nm nanodisks, respectively. The green circles show the average intensity of the molecules within that $20-\mathrm{nm}$ radial interval normalized divided by the average intensity of molecules at the actual position of the molecules (which is indicated by a black dashed line). The error bars indicate the standard deviation of the normalized molecule fluorescence intensities on each radial distance interval. Grey dashed line indicates no enhancement. 
(a)

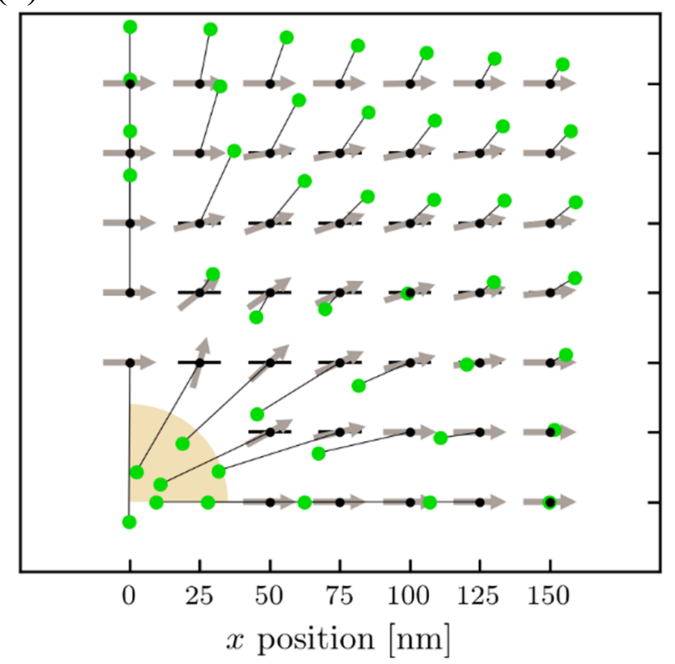

(c)

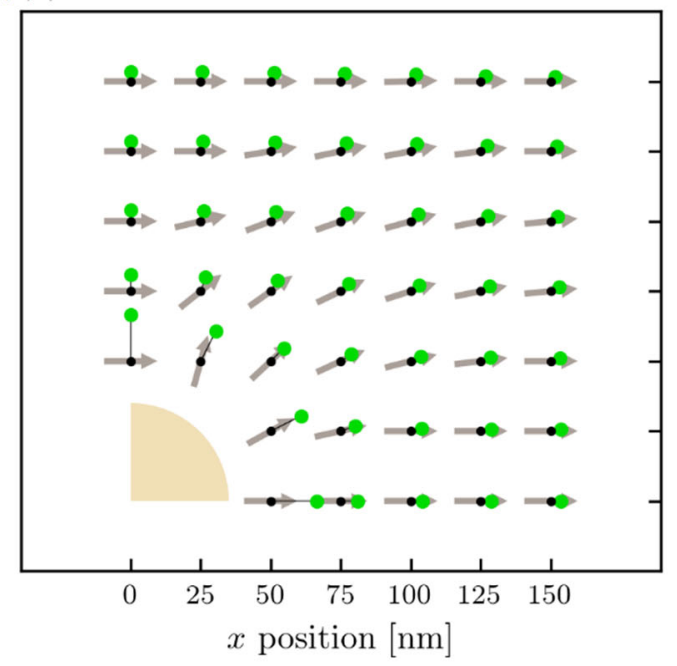

(b)

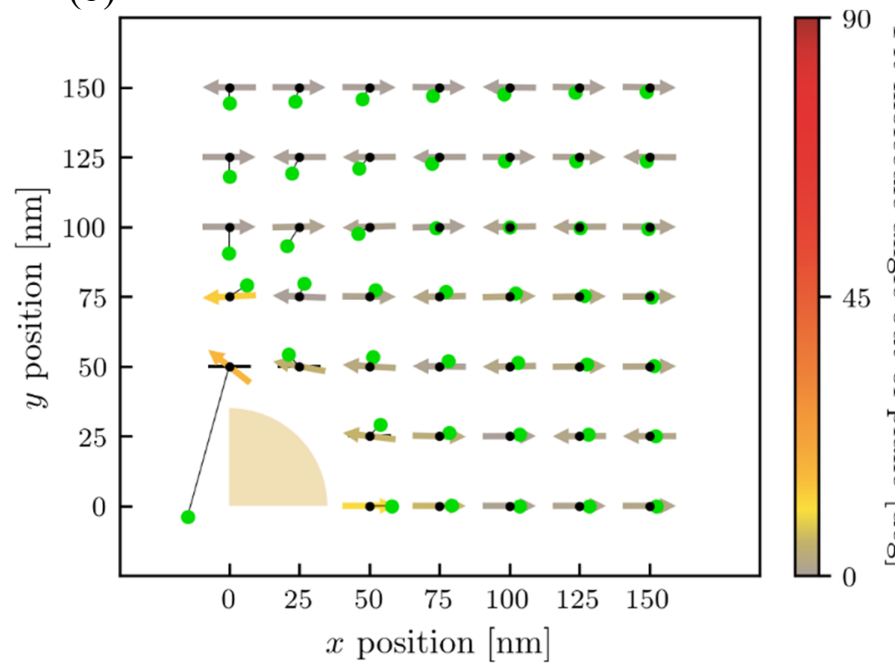

(d)

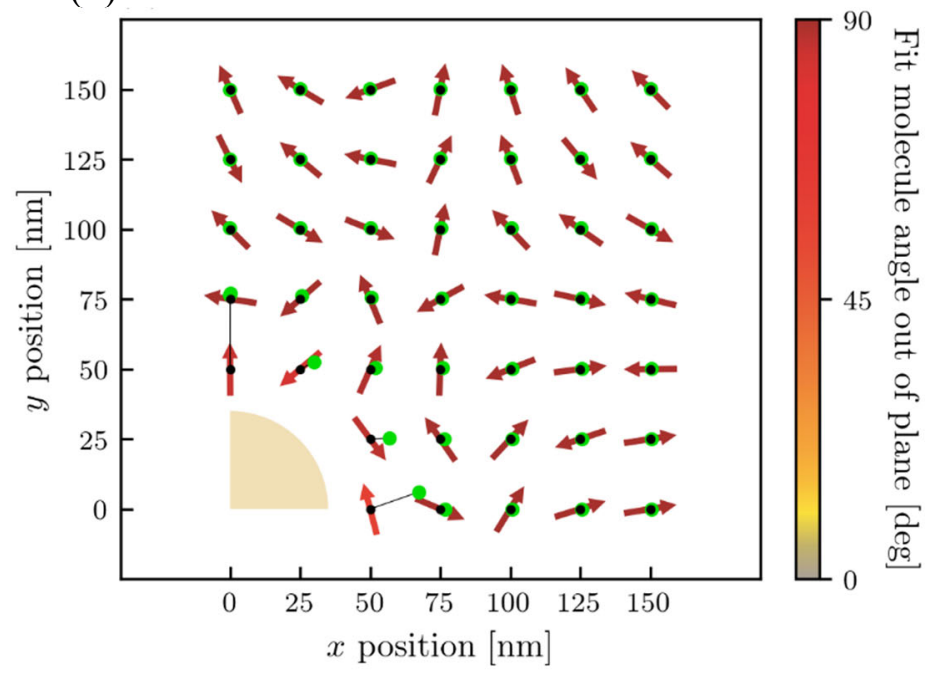

Figure S4. Fit positions for simulated dipole oscillators in the plane of an $h=36.82 \mathrm{~nm}$ and $d=$ $91.00 \mathrm{~nm}$ oblate spheroid parametrized to reproduce the scattering spectrum of the experimental 70-nm gold nanodisk. The black dots indicate the ground truth positions of molecular oscillators oriented in-plane along the $x$-axis (black horizontal lines, panels (a) and (b)) or out of the page (panels (c) and (d)). The green dots indicate the apparent emission position of each molecule after a fit to a Gaussian distribution ((a), (c)) or the coupled-dipole image model ((b), (d)), and the arrows indicate the predicted molecule orientation projected into the plane of the nanodisk with the color measuring its predicted out-of-plane projection. All images to fit were simulated at a higher resolution of $3.3 \mathrm{~nm} /$ pixel. 
(a)

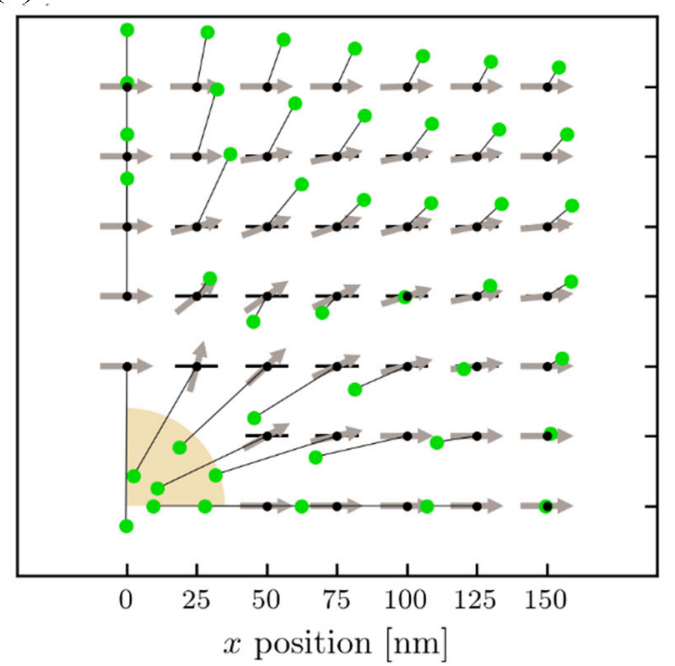

(c)

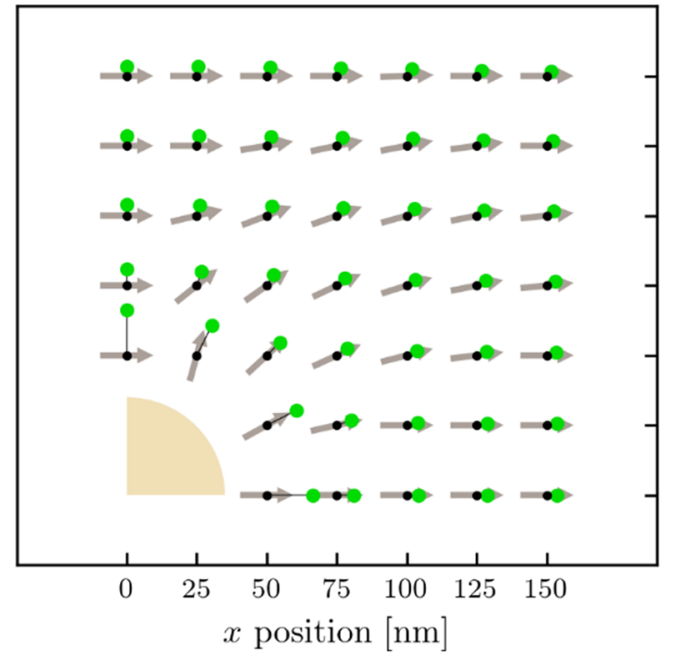

(b)

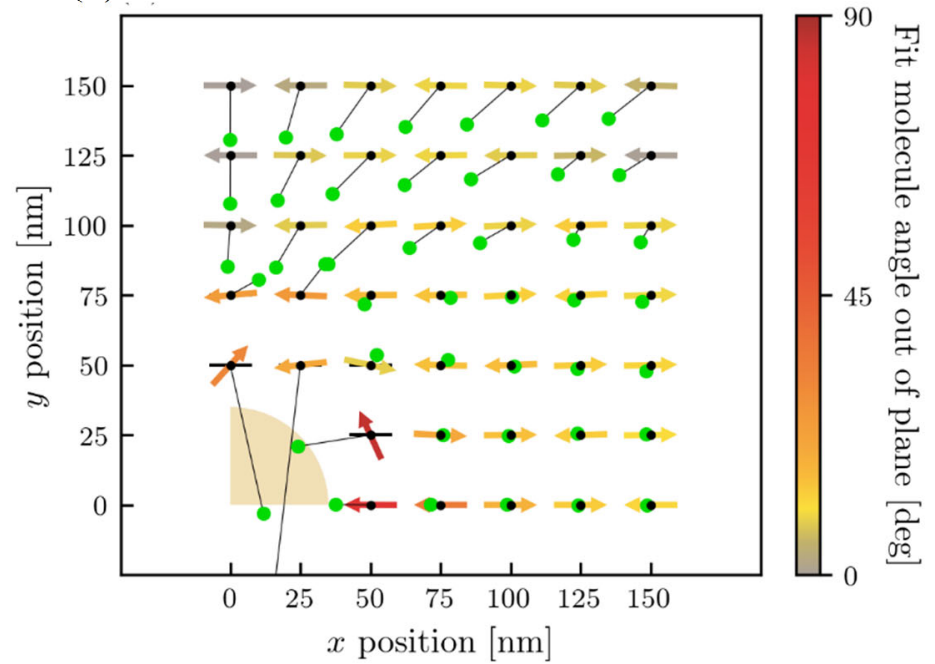

(d)

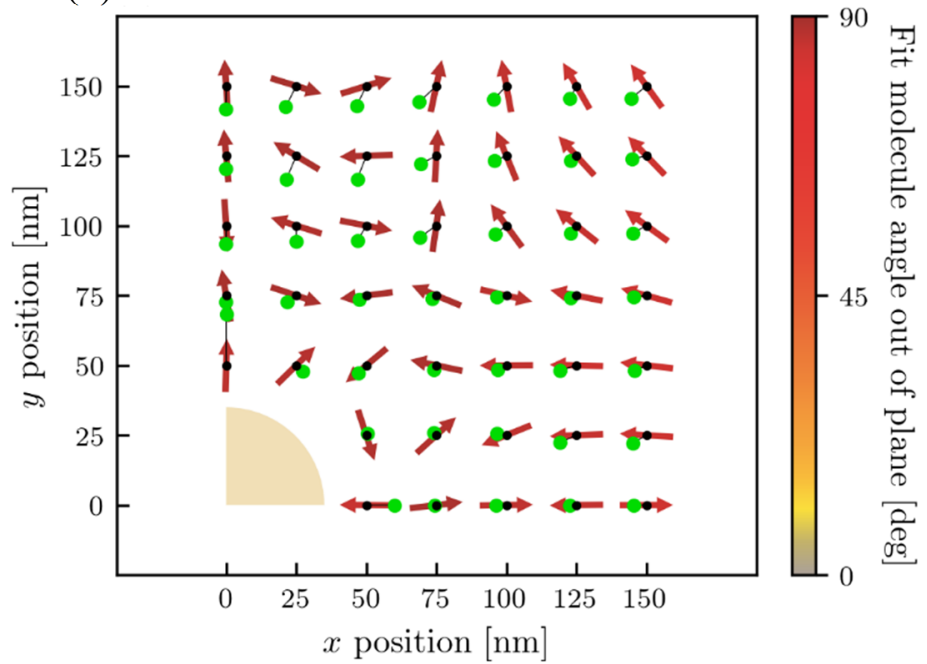

Figure S5. Fit positions for simulated dipole oscillators in the plane of an $h=36.82 \mathrm{~nm}$ and $d=$ $91.00 \mathrm{~nm}$ oblate spheroid parametrized to reproduce the scattering spectrum of the experimental 70-nm gold nanodisk. The black dots indicate the ground truth positions of molecular oscillators oriented in-plane along the $x$-axis (black horizontal lines, panels (a) and (b)) or out of the page (panels (c) and (d)). The green dots indicate the apparent emission position of each molecule after a fit to a Gaussian distribution ((a), (c)) or the coupled-dipole image model ((b), (d)), and the arrows indicate the predicted molecule orientation projected into the plane of the nanodisk with the color measuring its predicted out-of-plane projection. All images to fit were simulated at the lower resolution of $48.5 \mathrm{~nm} /$ pixel used in experiment. 
(a)

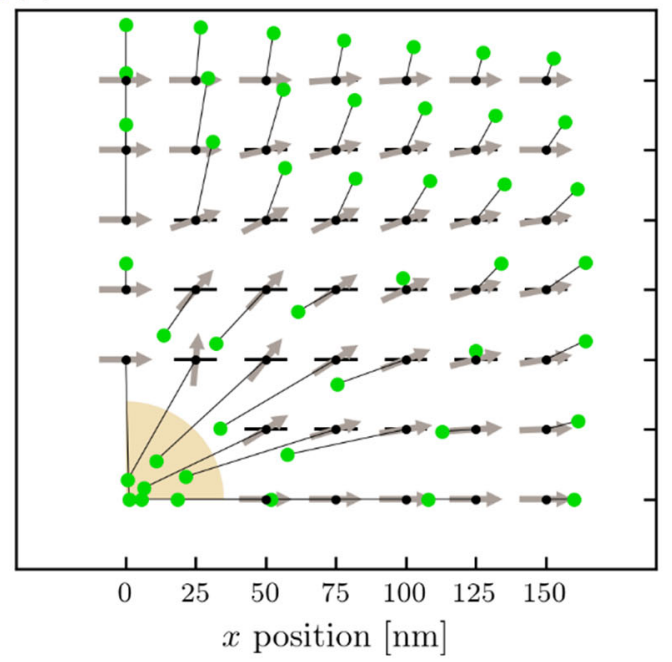

(c)

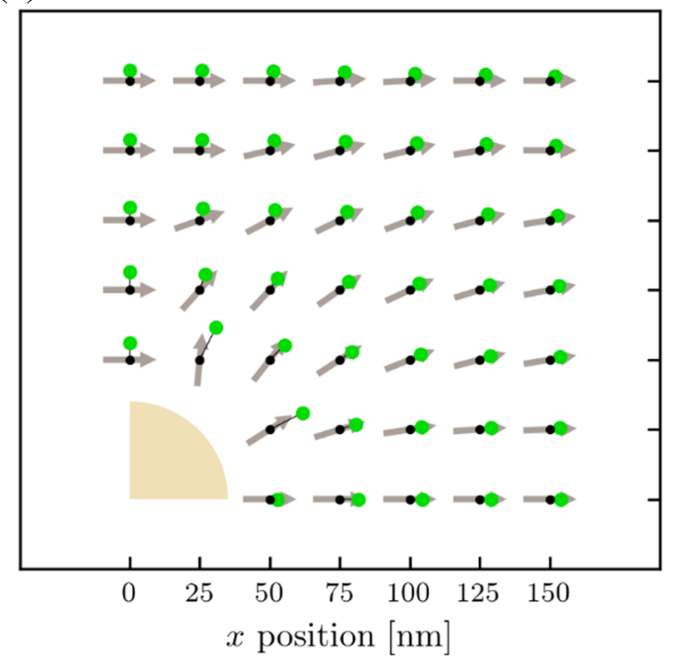

(b)

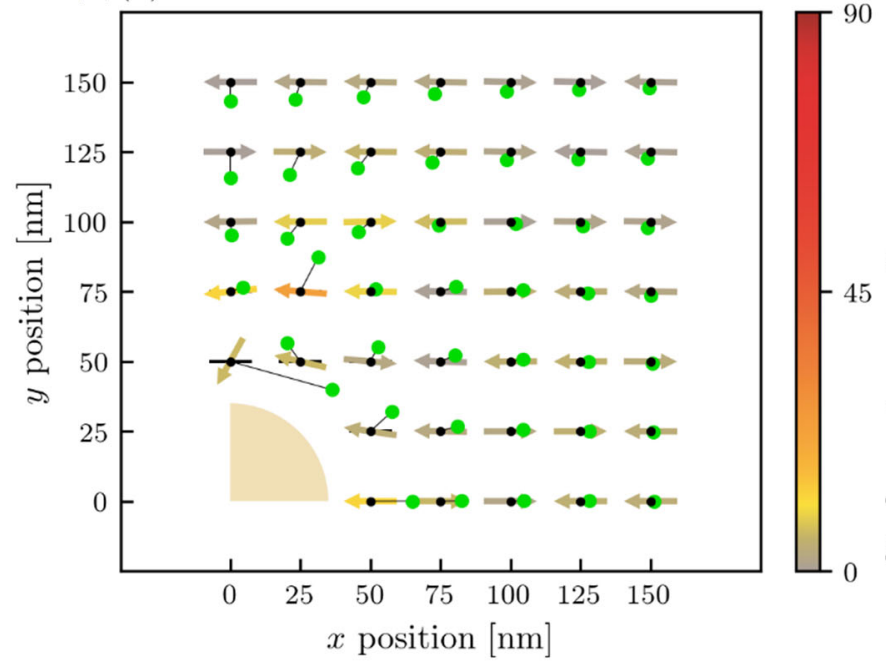

(d)

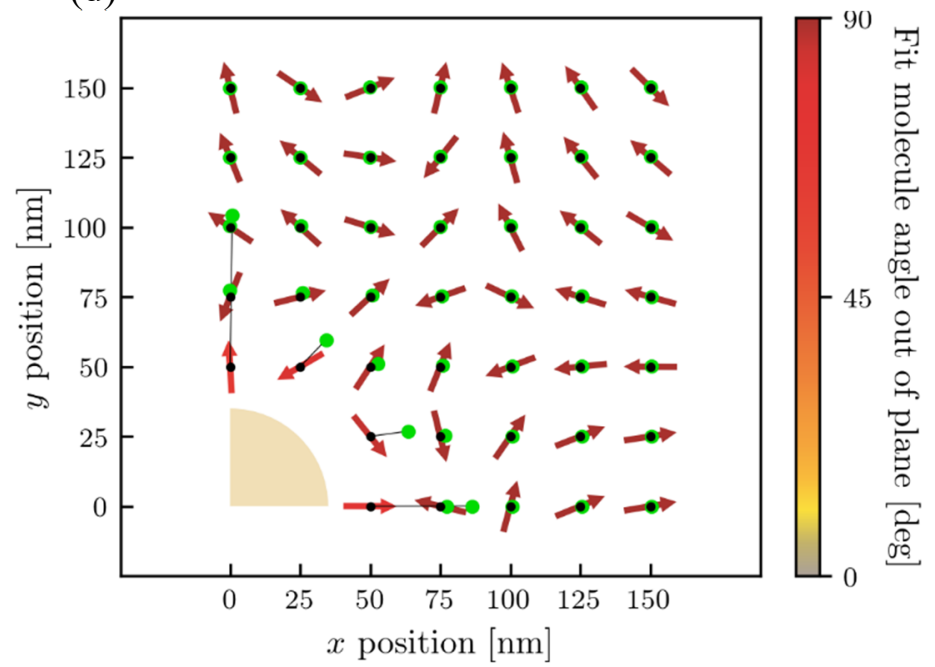

Figure S6. Fit positions for simulated dipole oscillators in the plane of an $h=36.82 \mathrm{~nm}$ and $d=$ $98.53 \mathrm{~nm}$ oblate spheroid parametrized to reproduce the scattering spectrum of the experimental 76-nm gold nanodisk. The black dots indicate the ground truth positions of molecular oscillators oriented in-plane along the $x$-axis (black horizontal lines, panels (a) and (b)) or out of the page (panels (c) and (d)). The green dots indicate the apparent emission position of each molecule after a fit to a Gaussian distribution ((a), (c)) or the coupled-dipole image model ((b), (d)), and the arrows indicate the predicted molecule orientation projected into the plane of the nanodisk with the color measuring its predicted out-of-plane projection. All images to fit were simulated at a higher resolution of $3.3 \mathrm{~nm} /$ pixel. 
(a)

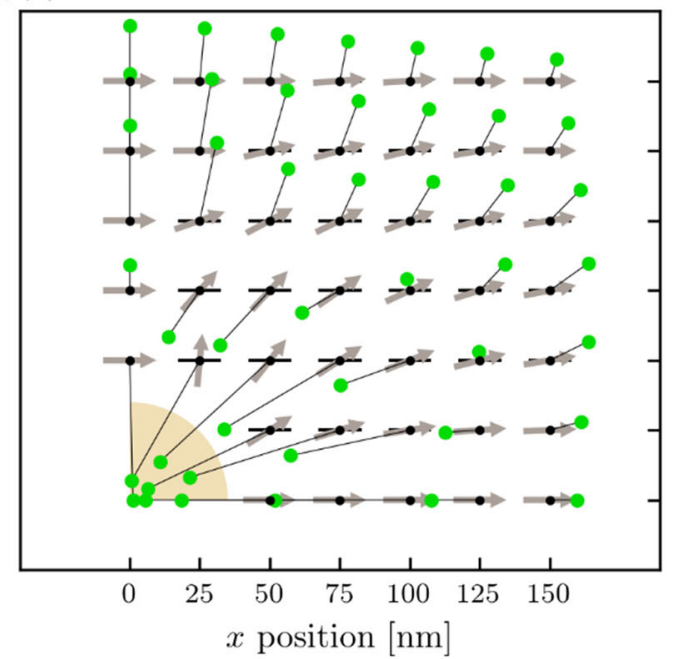

(c)

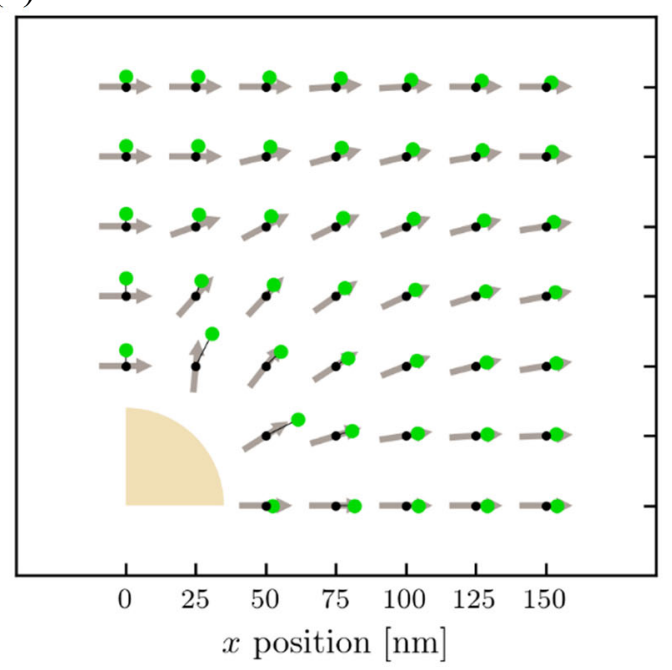

(b)

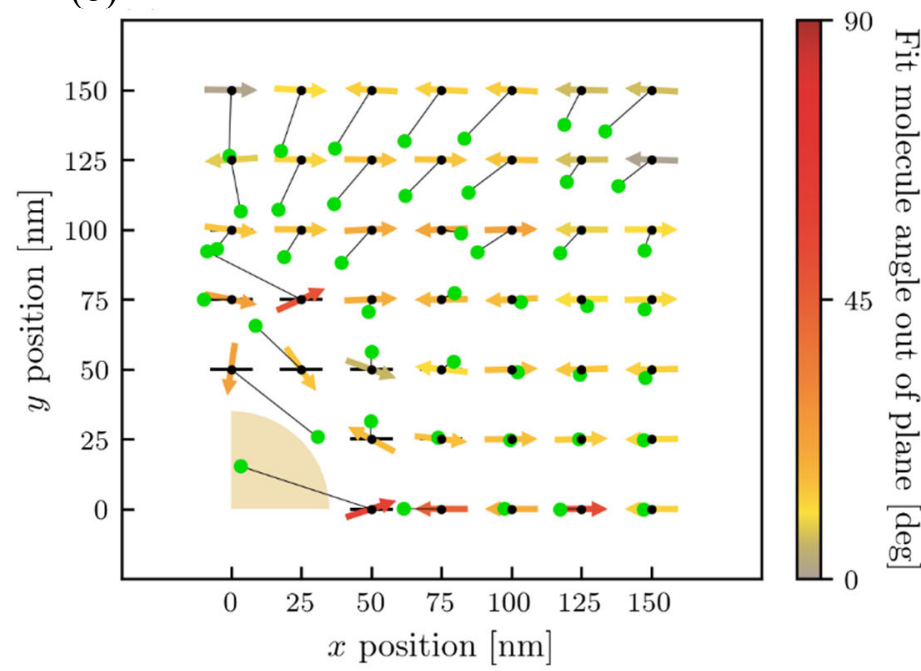

(d)

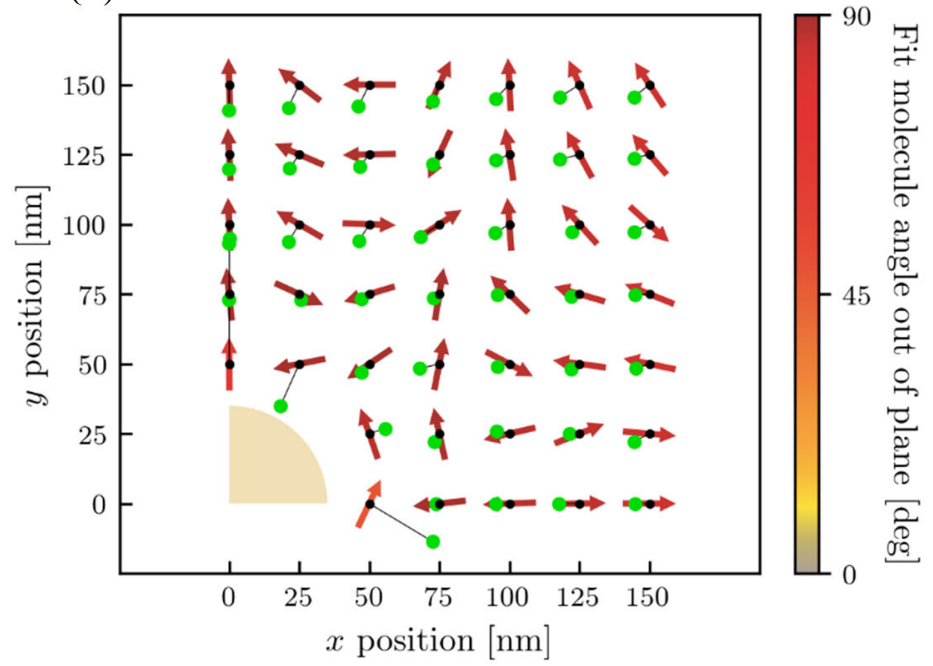

Figure S7. Fit positions for simulated dipole oscillators in the plane of an $h=36.82 \mathrm{~nm}$ and $d=$ $98.53 \mathrm{~nm}$ oblate spheroid parametrized to reproduce the scattering spectrum of the experimental 76-nm gold nanodisk. The black dots indicate the ground truth positions of molecular oscillators oriented in-plane along the $x$-axis (black horizontal lines, panels (a) and (b)) or out of the page (panels (c) and (d)). The green dots indicate the apparent emission position of each molecule after a fit to a Gaussian distribution ((a), (c)) or the coupled-dipole image model ((b), (d)), and the arrows indicate the predicted molecule orientation projected into the plane of the nanodisk with the color measuring its predicted out-of-plane projection. All images to fit were simulated at the lower resolution of $48.5 \mathrm{~nm} /$ pixel used in experiment. 
(a)

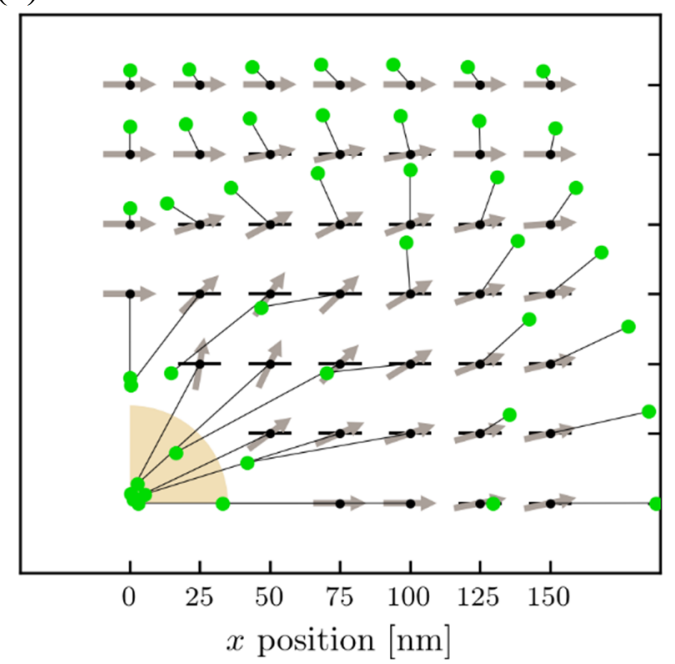

(c)

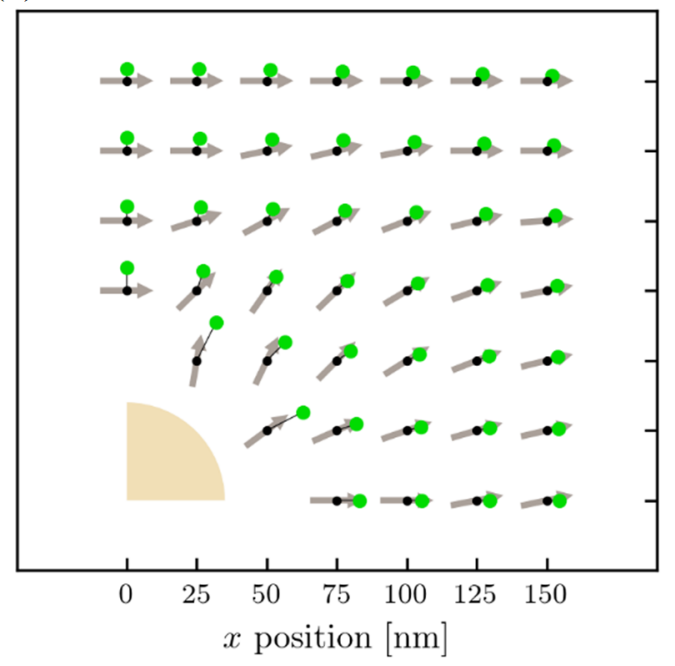

(b)

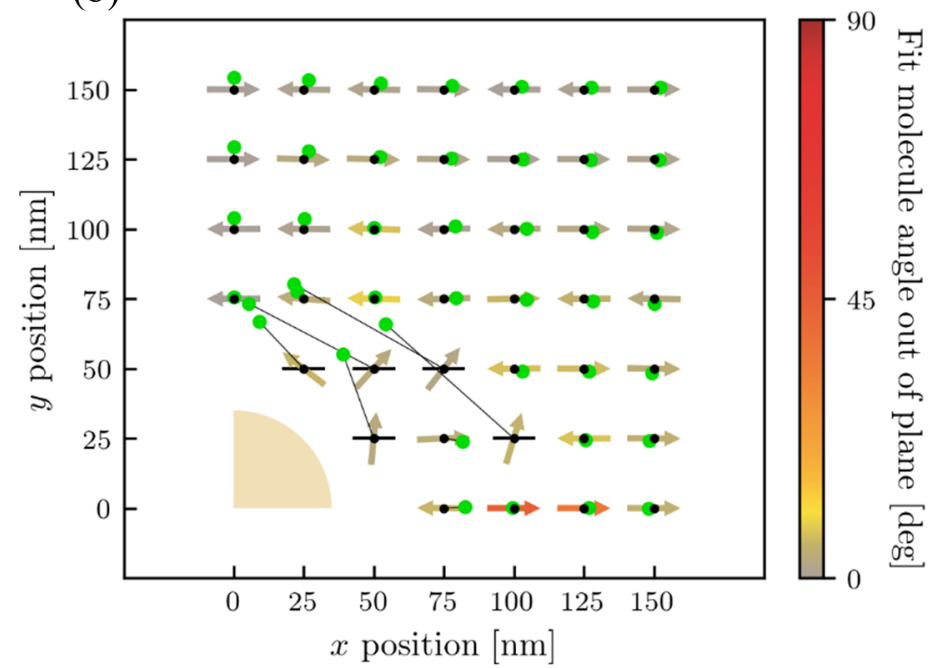

(d)

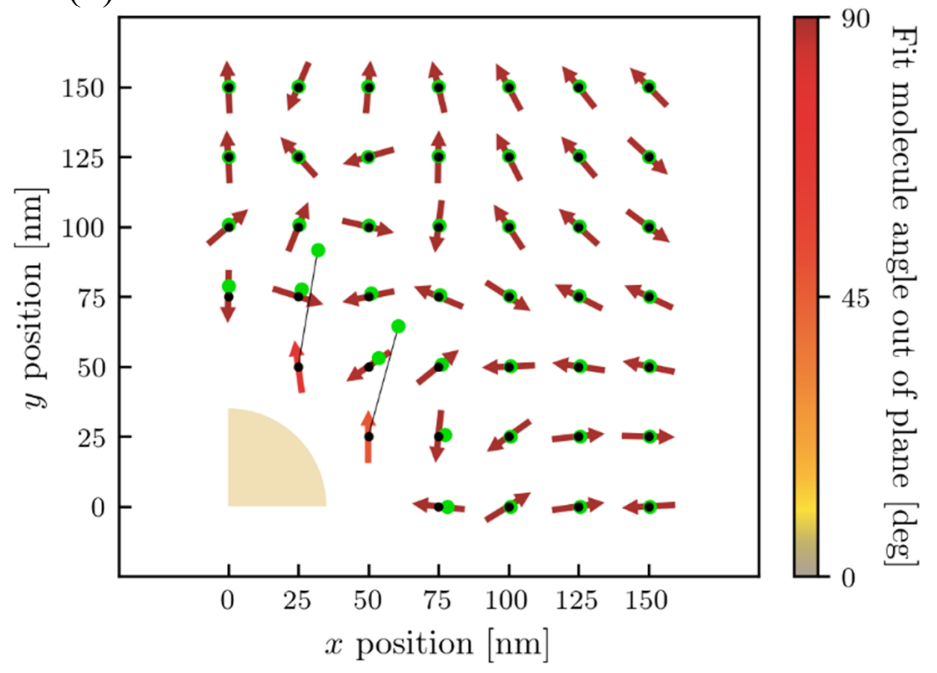

Figure S8. Fit positions for simulated dipole oscillators in the plane of an $h=36.82 \mathrm{~nm}$ and $d=$ $110.53 \mathrm{~nm}$ oblate spheroid parametrized to reproduce the scattering spectrum of the experimental 86-nm gold nanodisk. The black dots indicate the ground truth positions of molecular oscillators oriented in-plane along the $x$-axis (black horizontal lines, panels (a) and (b)) or out of the page (panels (c) and (d)). The green dots indicate the apparent emission position of each molecule after a fit to a Gaussian distribution ((a), (c)) or the coupled-dipole image model ((b), (d)), and the arrows indicate the predicted molecule orientation projected into the plane of the nanodisk with the color measuring its predicted out-of-plane projection. All images to fit were simulated at a higher resolution of $3.3 \mathrm{~nm} /$ pixel. 
(a)

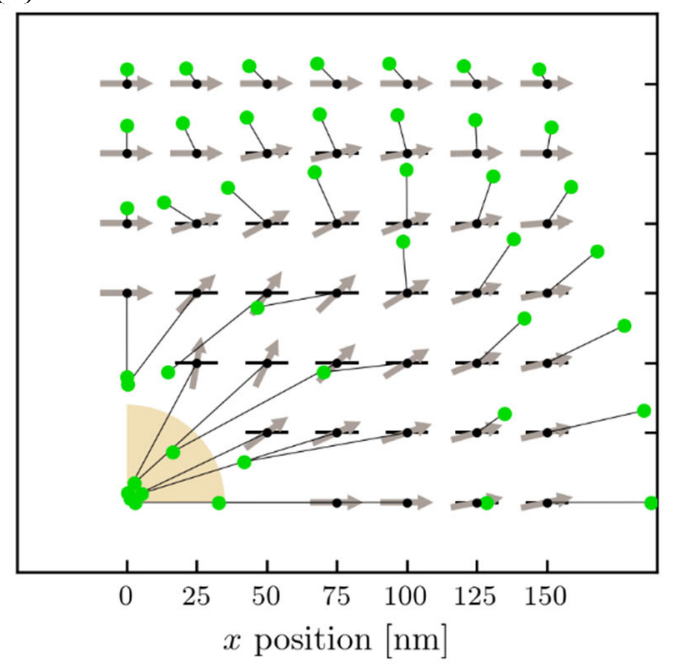

(c)

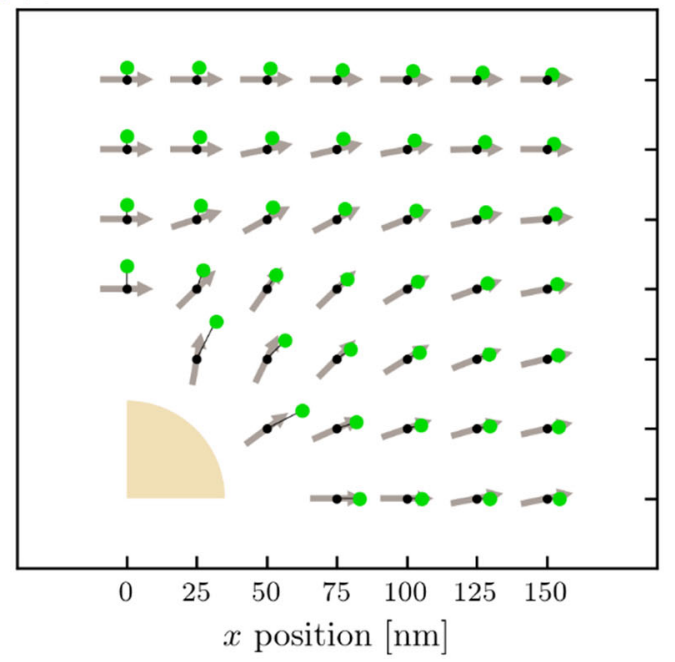

(b)

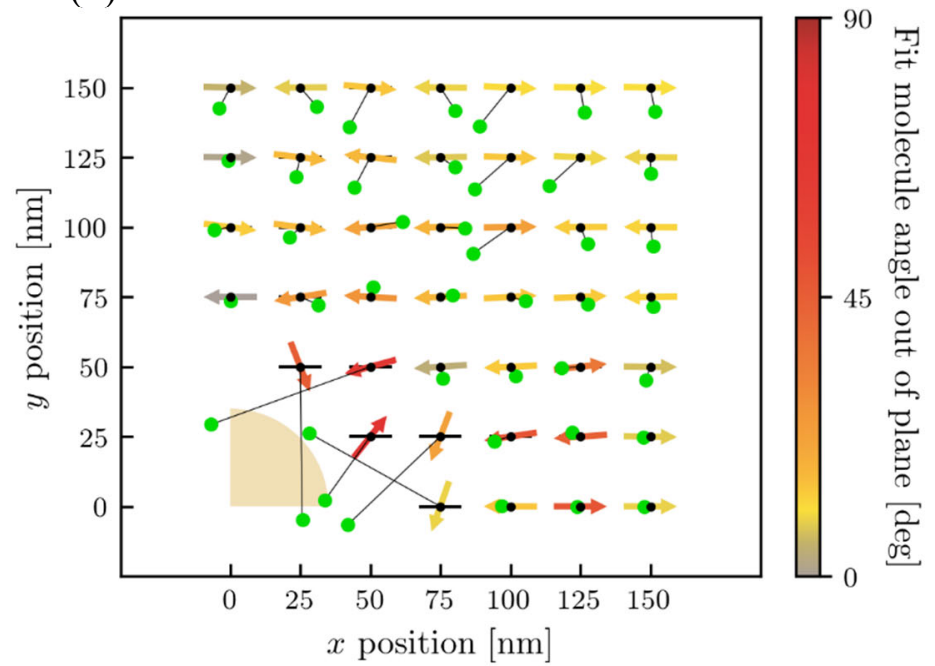

(d)

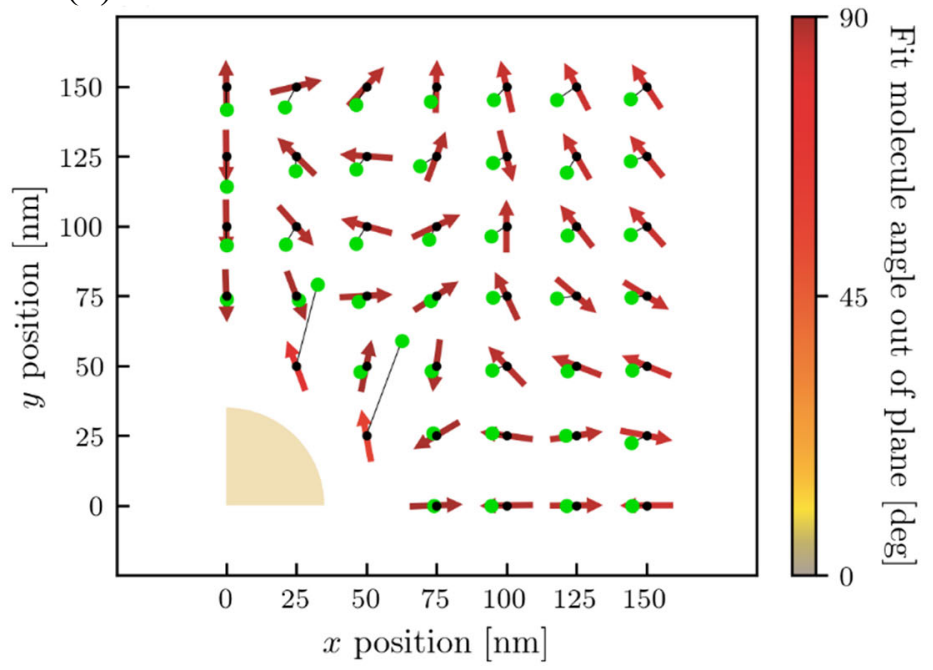

Figure S9. Fit positions for simulated dipole oscillators in the plane of an $h=36.82 \mathrm{~nm}$ and $d=$ $110.53 \mathrm{~nm}$ oblate spheroid parametrized to reproduce the scattering spectrum of the experimental 86-nm gold nanodisk. The black dots indicate the ground truth positions of molecular oscillators oriented in-plane along the $x$-axis (black horizontal lines, panels (a) and (b)) or out of the page (panels (c) and (d)). The green dots indicate the apparent emission position of each molecule after a fit to a Gaussian distribution ((a), (c)) or the coupled-dipole image model ((b), (d)), and the arrows indicate the predicted molecule orientation projected into the plane of the nanodisk with the color measuring its predicted out-of-plane projection. All images to fit were simulated at the lower resolution of $48.5 \mathrm{~nm} /$ pixel used in experiment. 
(a)

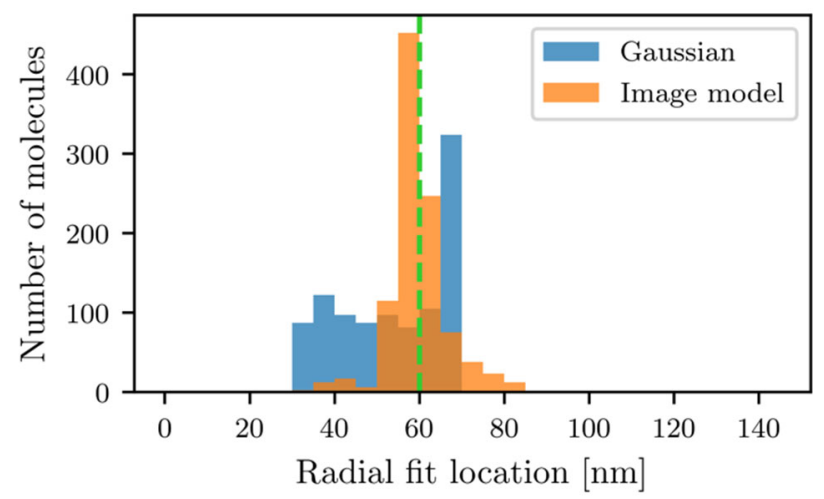

(b)

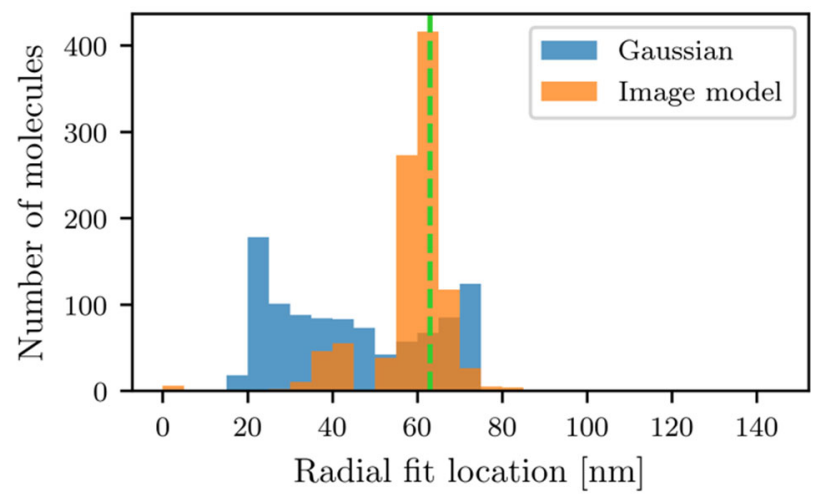

(c)

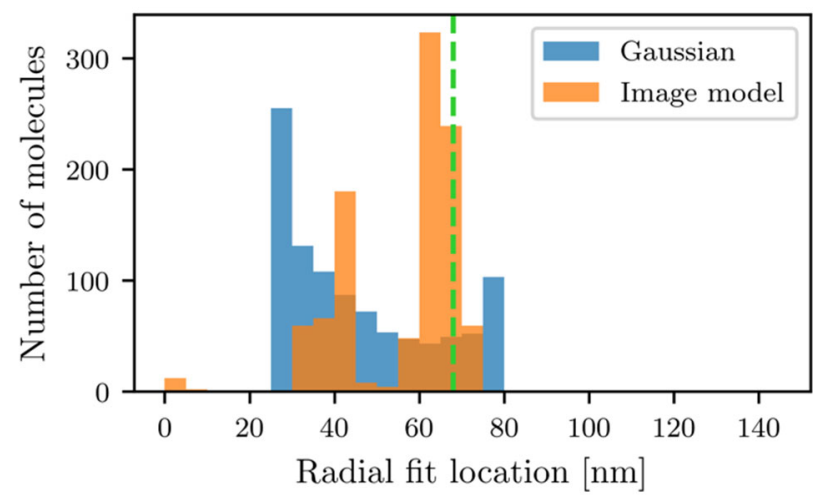

Figure S10. Histograms of radial fit locations of 1000 simulated ATTO590 dye molecules located $25 \mathrm{~nm}$ from a (a) 70-, (b) 76-, or (c) 86-nm gold nanodisk. The transition moment of all molecules is randomly oriented in the plane perpendicular to the DNA linker. Fits to a standard 2D Gaussian (blue) and to the image model (orange), result in the apparent radial localization density histograms for each fitting function. The green dashed lines indicate the actual radial position of the dye molecules in each assembly. 
(a)

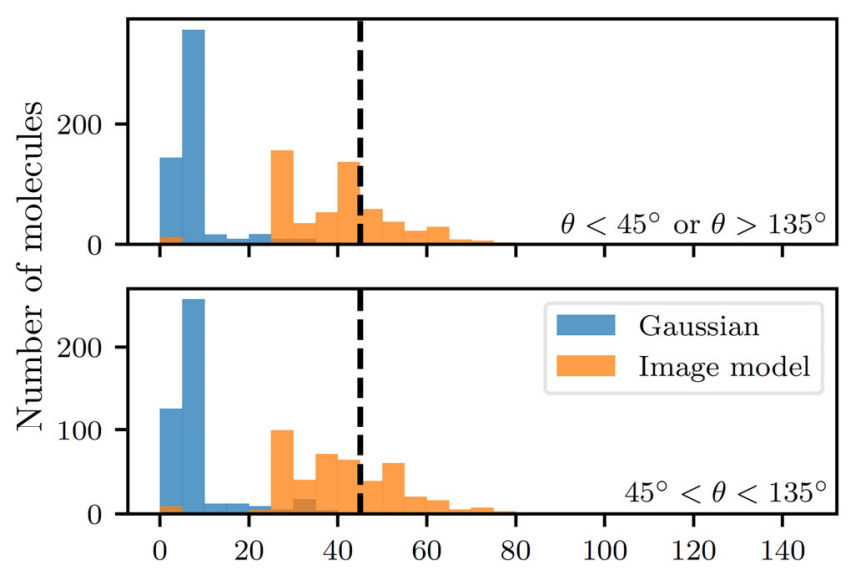

(b)

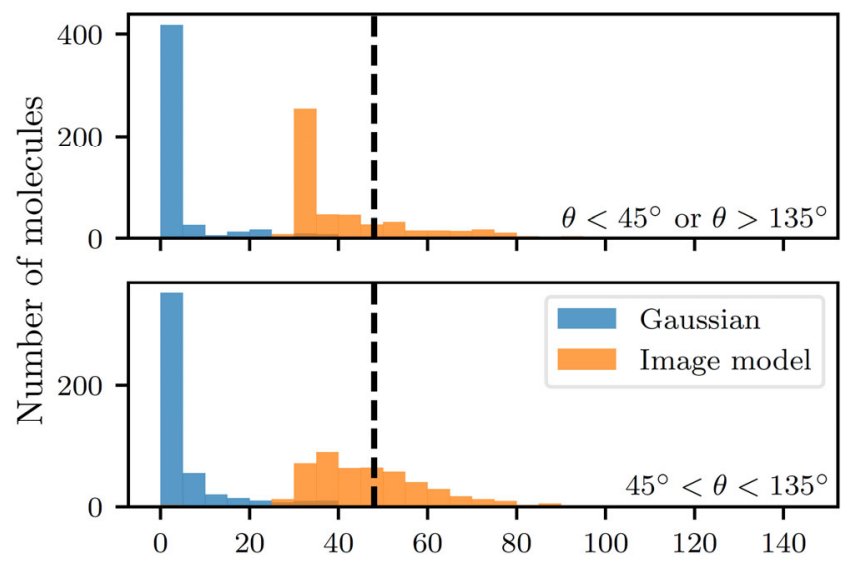

(c)

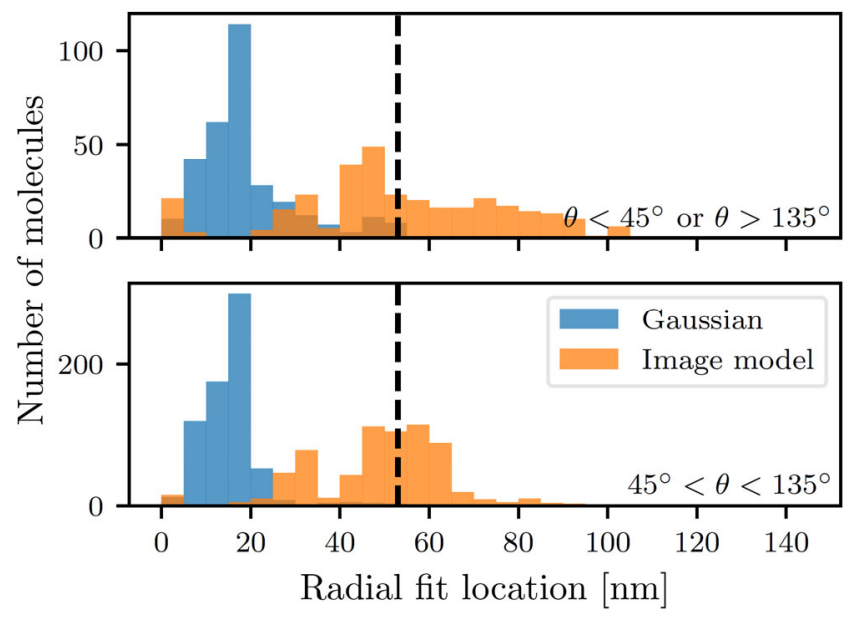

Figure S11. (a) - (c) Simulated images of single randomly oriented ATTO590 dye molecules coupled to 70-, 76-, and 86-nm oblate spheroids, respectively, are fit to a standard 2D Gaussian function (blue) and the coupled-dipole image model (orange) to derive the apparent radial localization density histograms for each fitting function (as in main text Fig. 5a-c). The black dashed lines indicate the actual radial position of the dye molecules in each assembly. Each collection is split into two panels: (top) molecules with polar angle, $\theta$, predicted to be $\leq 45^{\circ}$ or $\geq$ $135^{\circ}$, and (bottom) molecules with predicted polar angle $45^{\circ}<\theta<135^{\circ}$. 
(a)

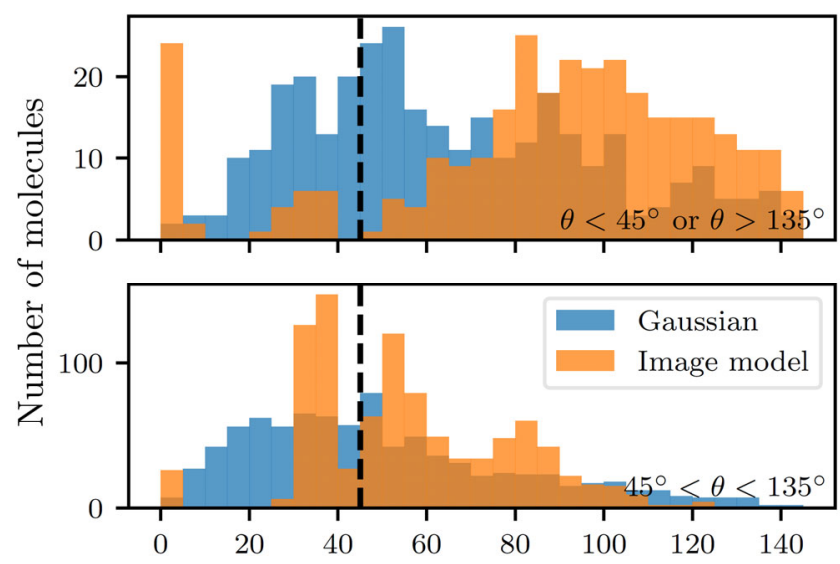

(b)

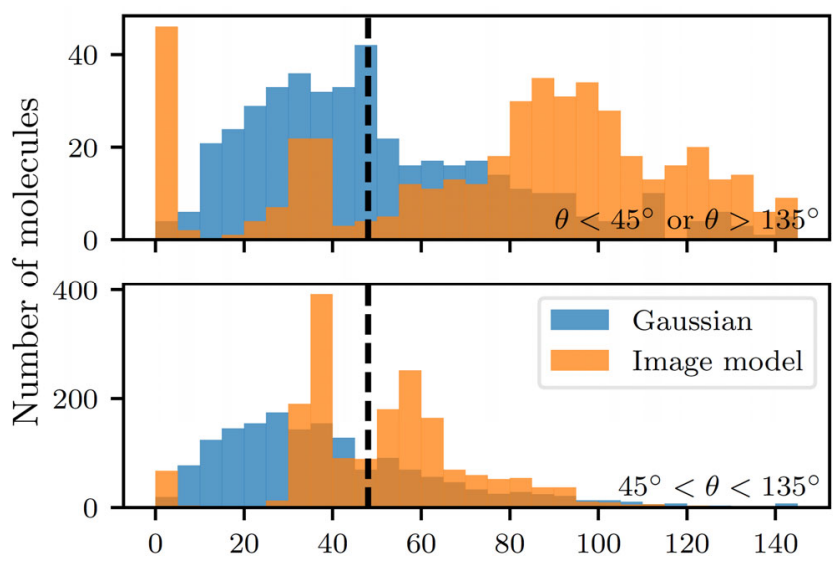

(c)

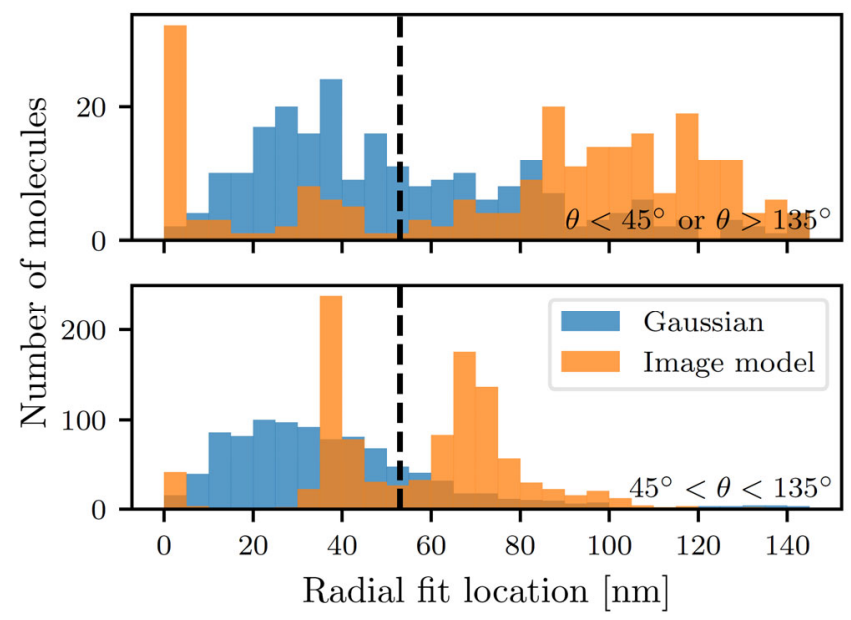

Figure S12. (a) - (c) Experimental images of single ATTO590 dye molecules coupled to 70-, 76-, and 86-nm oblate spheroids, respectively, are fit to a standard 2D Gaussian function (blue) and the coupled-dipole image model (orange) to derive the apparent radial localization density histograms for each fitting function (as in main text Fig. 5d-f). The black dashed lines indicate the actual radial position of the dye molecules in each assembly. Each collection is split into two panels: (top) molecules with polar angle, $\theta$, predicted to be $\leq 45^{\circ}$ or $\geq 135^{\circ}$, and (bottom) molecules with predicted polar angle $45^{\circ}<\theta<135^{\circ}$. 
(a)

$\mathrm{r}_{\mathrm{fit}}<50 \mathrm{~nm}$
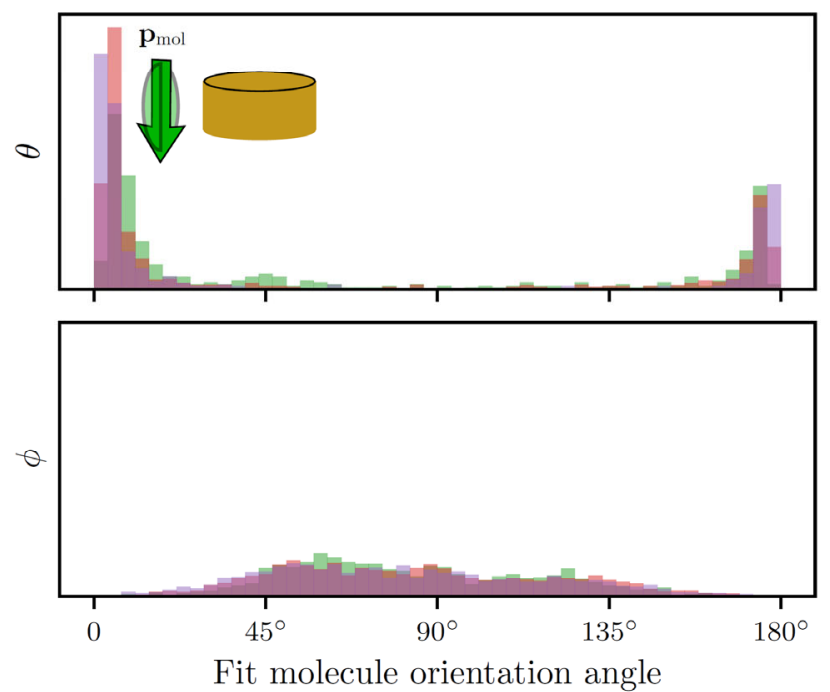

(b)
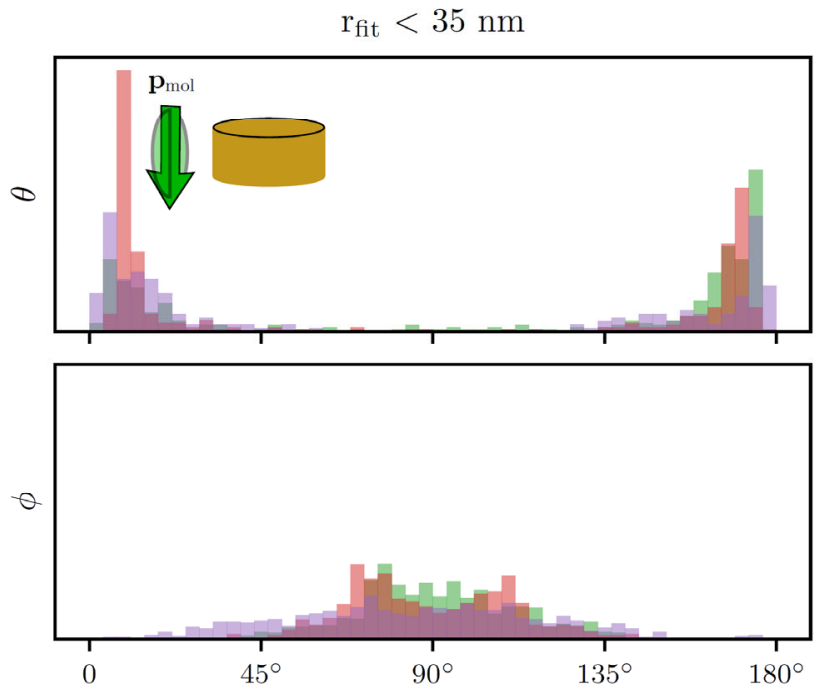

Fit molecule orientation angle $\mathrm{r}_{\text {fit }}>50 \mathrm{~nm}$
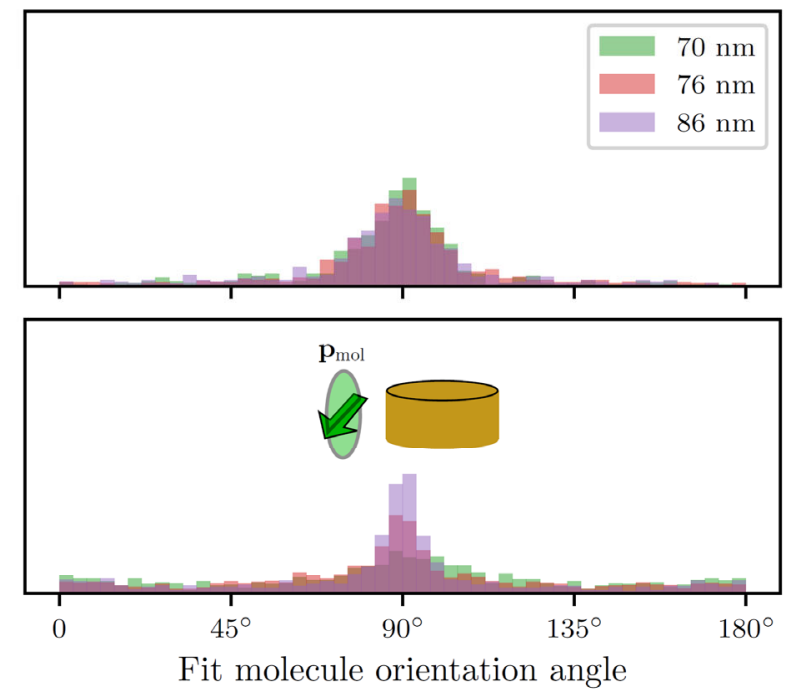

$\mathrm{r}_{\text {fit }}>35 \mathrm{~nm}$
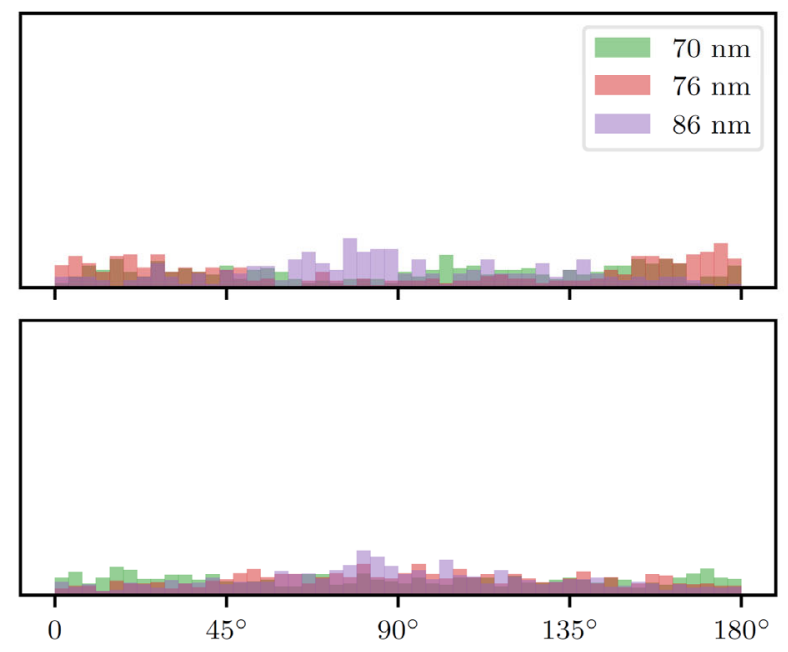

Fit molecule orientation angle

Figure S13. Model-predicted dye orientations in (a) experiments and (b) simulations. Most molecules are predicted to be oriented in the plane perpendicular to the radial DNA for both fits to experimental and simulated image, with image fit bifurcating naturally into molecules predicted to be too close to the disk (left column) and molecules predicted too far from the disk (right column). The molecules predicted to be too close are consistently assigned by the model an orientation out of the plane, or along the optical axis. Meanwhile, the rest of the experimental molecules are assigned to orient close to the focal plane $\left(\theta=90^{\circ}\right)$ and perpendicular to the radially-directed DNA $\left(\varphi=90^{\circ}\right)$. In simulation, this effect is less clear, but so is the distinction between the two groups of molecules (see main text Fig. 5). 
(a)

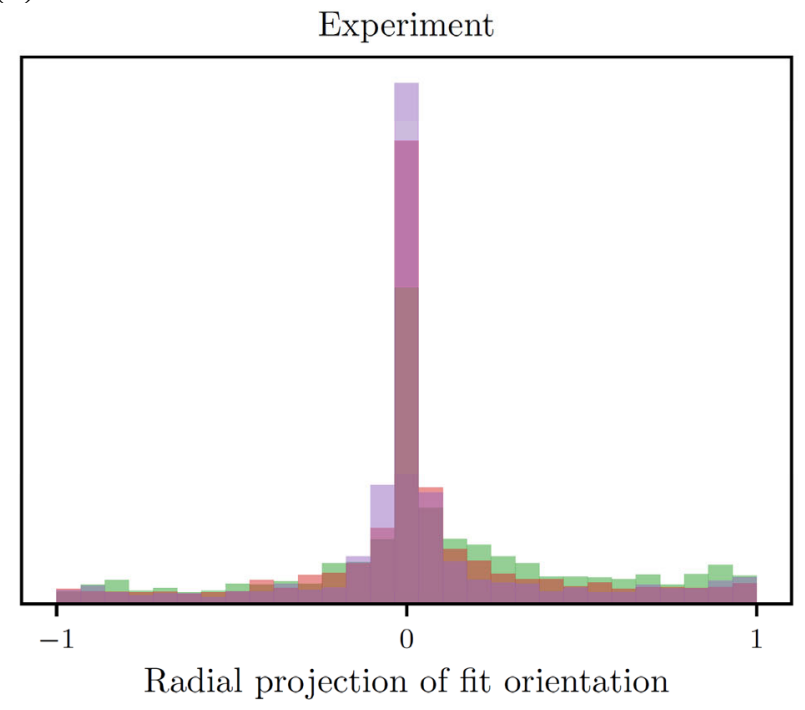

(b)

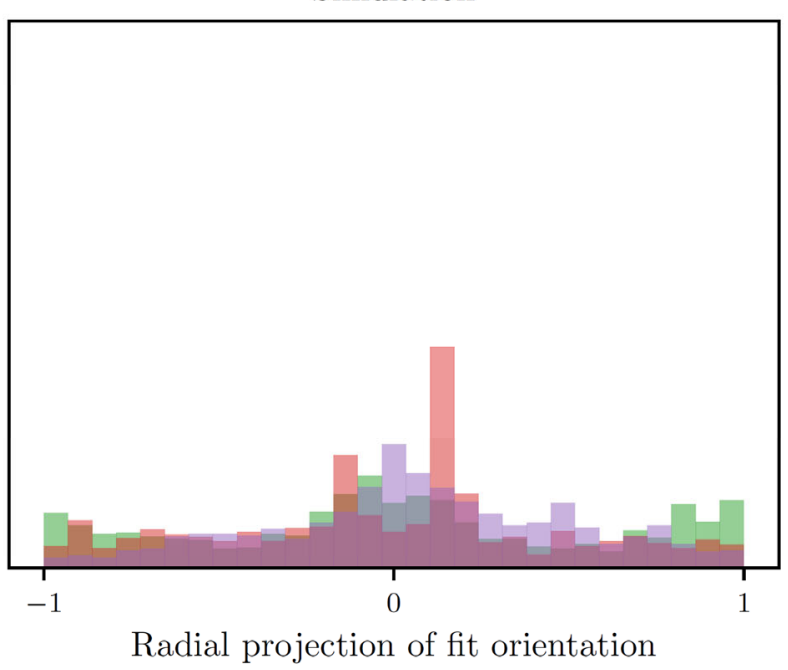

Figure S14. Fit dye orientations projected onto the DNA linker axis. Most molecules are predicted to be oriented in the plane perpendicular to the radial DNA for both fits to (a) experimental and (b) simulated images. The distributions are noticeably flatter for the simulated images (especially for the 76-nm nanodisk in green), which indicates interference from the higher-order plasmon modes. The relative sharpness of the experimental distributions may be due to weakening of the quadrupole response caused by features of the experiment not accounted for in simulations (e.g., the substrate, DNA, and silicon dioxide cap). Green: 70-nm nanodisks; Pink: 76-nm nanodisks; Purple: 86-nm nanodisks. 
Table S1. Oligonucleotides used in this study.

\begin{tabular}{|c|c|}
\hline $\begin{array}{l}\text { Number of DNA } \\
\text { base pairs }\end{array}$ & Sequence \\
\hline 30 & $\begin{array}{l}\text { 5- /5ThioMC6-D/CTT GCC ATC GCT AGT ACA TGG CAC } \\
\text { TGA CTG -3 }\end{array}$ \\
\hline 30 & $\begin{array}{l}\text { 5- /5ATTO590N/CAG TCA GTG CCA TGT ACT AGC GAT } \\
\text { GGC AAG -3 }\end{array}$ \\
\hline
\end{tabular}

